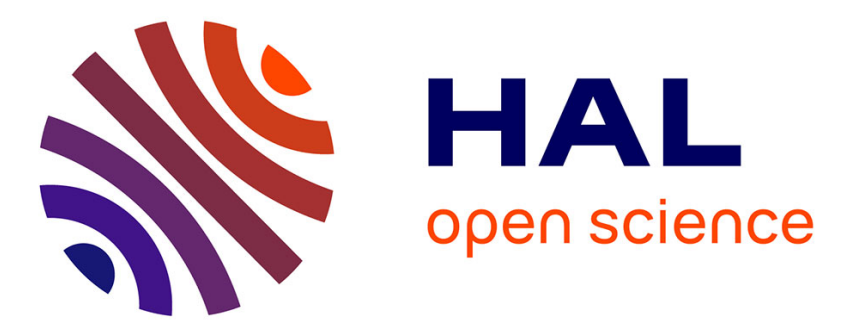

\title{
Comportement micromécanique d'un verre métallique Fe-B-Si-C chargé en hydrogène
}

\author{
J.P. Allemand, F. Fouquet, J. Perez
}

\section{To cite this version:}

J.P. Allemand, F. Fouquet, J. Perez. Comportement micromécanique d'un verre métallique Fe-B-Si-C chargé en hydrogène. Revue de Physique Appliquée, 1986, 21 (1), pp.1-9. 10.1051/rphysap:019860021010100 . jpa-00245406

\section{HAL Id: jpa-00245406 https://hal.science/jpa-00245406}

Submitted on 1 Jan 1986

HAL is a multi-disciplinary open access archive for the deposit and dissemination of scientific research documents, whether they are published or not. The documents may come from teaching and research institutions in France or abroad, or from public or private research centers.
L'archive ouverte pluridisciplinaire HAL, est destinée au dépôt et à la diffusion de documents scientifiques de niveau recherche, publiés ou non, émanant des établissements d'enseignement et de recherche français ou étrangers, des laboratoires publics ou privés. 


\title{
REVUE DE PHYSIQUE APPLIQUÉE
}

Revue Phys. Appl. 21 (1986) 1-9

JANVIER 1986, PAGE 1

Classification

Physics Abstracts

$61.40 \mathrm{D}-62.40-75.50 \mathrm{~K}-75.80$

\section{Comportement micromécanique d'un verre métallique Fe-B-Si-C chargé en hydrogène}

\author{
J. P. Allemand, F. Fouquet et J. Perez \\ Groupe d'Etudes de Métallurgie Physique et de Physique des Matériaux, \\ Laboratoire associé au C.N.R.S. - 341, Bât. 502, I.N.S.A., 20, avenue A. Einstein, \\ 69621 Villeurbanne Cedex, France
}

(Reçu le 19 avril 1985, révisé le 2 septembre, accepté le 23 septembre 1985)

\begin{abstract}
Résumé. - Les verres métalliques ferromagnétiques et magnétostrictifs soumis à une contrainte cyclique sont le siège d'un effet magnétoélastique, ou effet $\Delta E$. En frottement intérieur, cet effet se caractérise par un amortissement d'ordre magnétomécanique. Ce comportement micromécanique a été suivi dans le cas d'un verre métallique base fer $\left(\mathrm{Fe}_{83} \mathrm{~B}_{14} \mathrm{Si}_{1,5} \mathrm{C}_{1,5}\right)$ en relation avec l'état structural du matériau. Par ailleurs, le chargement en hydrogène par voie électrolytique sur un tel matériau entraîne la mise en évidence d'un pic de frottement intérieur dont l'origine physique ne peut être uniquement liée au seul phénomène relaxationnel connu sous le nom de pic de Snoek. Il ressort de cette étude l'existence d'une interaction importante entre les parois de domaines ferromagnétiques et les atomes d'hydrogène ainsi que d'une redistribution ou réorientation des atomes d'hydrogène sous l'effet d'un champ magnétique, liée à la nature magnétostrictive du matériau.
\end{abstract}

\begin{abstract}
When a cyclic stress is applied, ferromagnetic and magnetostrictive metallic glasses exhibit a magnetoelastic effect or $\Delta E$-effect. Such an effect is associated to a magnetomechanical damping. This micromechanical behaviour has been studied in the case of an iron-based amorphous alloy $\left(\mathrm{Fe}_{83} \mathrm{~B}_{14} \mathrm{Si}_{1.5} \mathrm{C}_{1.5}\right)$, as a function of the structural state of the material. On the other hand, after electrolytically introducing hydrogen, an internal friction peak is observed. The physical processus leading to such a peak cannot only be attributed to the relaxational phenomenon well known as Snoek peak. A strong interaction between ferromagnetic domain walls and hydrogen atoms and a redistribution or a reorientation of hydrogen atoms under magnetic field, due to the magnetostrictive property of the material, are suggested in order to explain all the experimental features.
\end{abstract}

\section{Introduction.}

Le but de cette étude est de présenter le comportement micromécanique d'un verre métallique ferromagnétique et magnétostrictif, chargé en hydrogène, en relation avec l'état structural du matériau.

L'étude des propriétés micromécaniques des verres métalliques par mesures combinées du module d'Young et du frottement intérieur s'est avérée être, depuis quelques années, d'un grand intérêt, ces deux grandeurs physiques étant particulièrement sensibles, entre autres, à la relaxation structurale [1-7]. Dans le cas d'un matériau non ferromagnétique, la relaxation structurale a pour conséquence une augmentation du module, liée essentiellement à la densification du matériau et une baisse du frottement intérieur, résultant de la diminution de la mobilité des atomes.

Pour un verre métallique ferromagnétique et magnétostrictif, tel que le $\mathrm{FeBSiC}$, le traitement thermique conduisant à la relaxation structurale induit un relâchement des contraintes internes créées lors de l'élaboration par hypertrempe, ce qui facilite le mouvement des parois des domaines ferromagnétiques sous l'effet d'une contrainte appliquée [8]. Dans un tel matériau, la relaxation structurale aura ainsi pour conséquence le développement d'une déformation d'ordre magnétostrictif, à l'origine de l'effet $\Delta E$ ou effet magnétoélastique qui se traduit par une diminution du module d'Young [9].

L'effet magnétoélastique dépend de la composition 
du matériau par l'intermédiaire du coefficient de magnétostriction et de l'état structural par le biais des contraintes internes. Il est également fonction de la température [7], de l'aimantation du matériau, de l'amplitude et de la fréquence de la sollicitation mécanique [10].

Le couplage magnétomécanique s'accompagne également d'une augmentation du frottement intérieur résultant de la composante magnétostrictive de la déformation en quadrature avec la contrainte appliquée.

Par ailleurs, pour les verres métalliques chargés en hydrogène, il apparaît un pic de frottement intérieur $[11,12]$ couramment attribué à une réorientation, sous l'effet de la contrainte, des dipôles élastiques que forment les atomes d'hydrogène (analogie avec l'effet Snoek dans les matériaux cristallins). Pour des verres métalliques ferromagnétiques et magnétostrictifs, Berry et Pritchet ont montré, par mesure de frottement intérieur, une forte interaction entre les parois de Bloch et les atomes d'hydrogène [13]. Ce piégeage par les atomes d'hydrogène a également été envisagé par Chambron et al. [14] : l'existence d'une anisotropie magnétique serait induite localement par une redistribution des atomes d'hydrogène en positions interstitielles.

Au cours de la présente étude, nous allons tout d'abord suivre l'évolution des propriétés micromécaniques en liaison avec l'état structural d'un alliage FeBSiC (états brut d'élaboration et relaxé structuralement) afin de déterminer l'influence de cet état sur les variations de l'effet $\Delta E$ et de l'amortissement magnétomécanique. Dans un second temps, nous étudierons les mêmes échantillons chargés en hydrogène et nous montrerons quelle est l'incidence de cet hydrogène (et en particulier du couplage hydrogène-parois) sur le comportement micromécanique du matériau.

\section{Conditions expérimentales.}

Les rubans, de composition atomique $\mathrm{Fe}_{83}$ $\mathrm{B}_{14} \mathrm{Si}_{1,5} \mathrm{C}_{1,5}$ ont été élaborés par hypertrempe depuis l'état liquide au Centre de Recherches de Pont-àMousson. Les mesures de module d'Young et de frottement intérieur sont effectuées sur un anélasticimètre en utilisant la méthode de la lame vibrante. Les échantillons de dimensions utiles $12 \times 1,1 \times$ $0,035 \mathrm{~mm}^{3}$ sont placés dans une enceinte sous un vide de $10^{-2}$ à $10^{-3}$.torr; l'excitation et la détection de la vibration se font par voie électrostatique [7]. Les fréquences de vibration utilisées correspondent au mode fondamental $(f \simeq 150 \mathrm{~Hz})$ et au premier harmonique $(f \simeq 1000 \mathrm{~Hz})$.

Un champ magnétique de 0 à $6000 \mathrm{~A} / \mathrm{m}$ peut être appliqué dans le sens longitudinal de l'éprouvette à l'aide de deux bobines d'Helmholtz situées à l'intérieur de l'enceinte.

Les mesures peuvent être effectuées entre 80 et $720 \mathrm{~K}$. Le chauffage est réalisé par l'intermédiaire d'un four placé dans l'enceinte et d'une régulation programmation assurant, dans le cas présent, une vitesse de montée en température de $1 \mathrm{~K} / \mathrm{min}$. Le refroidissement à $80 \mathrm{~K}$ est obtenu en plongeant l'enceinte dans un cryostat contenant de l'azote liquide.

Le chargement en hydrogène se fait par voie électrolytique dans une solution d' $\mathrm{H}_{2} \mathrm{SO}_{4} 1 \mathrm{~N}$ contenant quelques p.p.m. de $\mathrm{CS}_{2}$ afin d'empêcher la recombinaison des atomes d'hydrogène à la surface de l'échantillon avant l'absorption par le matériau. La densité de courant est de environ $100 \mathrm{~mA} / \mathrm{cm}^{2}$. Le temps de chargement étant de $15 \mathrm{~min}$, cela correspond pour ce type d'alliage à une concentration en hydrogène comprise entre 1 et $2 \%$ atomique.

Sur les courbes expérimentales qui vont maintenant être présentées, les valeurs du module sont ramenées à la valeur $E_{0}$, représentant le module du matériau brut d'élaboration mesuré à $300 \mathrm{~K}$, avant tout traitement.

\section{Résultats expérimentaux.}

3.1 Etat STRUCtural ET COUPlage MagnétoÉLASTIQUE. - Nous allons préciser ici la différence de comportement micromécanique entre un échantillon brut d'élaboration et un échantillon relaxé structuralement. La relaxation structurale de l'alliage a été effectuée in situ sur l'installation de mesure en portant l'échantillon à une température de $620 \mathrm{~K}$.

Les résultats obtenus sont présentés surr les figures 1 et 2 . Les courbes a et $a^{\prime}$ de la figure 1 relatives à un échantillon brut d'élaboration font apparaître, par différence entre l'état aimanté et l'état désaimanté, un léger effet $\Delta E$ de l'ordre de 4 à $5 \%$ à $300 \mathrm{~K}$, ce qui signifierait que le niveau des contraintes internes induites lors de l'élaboration par hypertrempe n'est

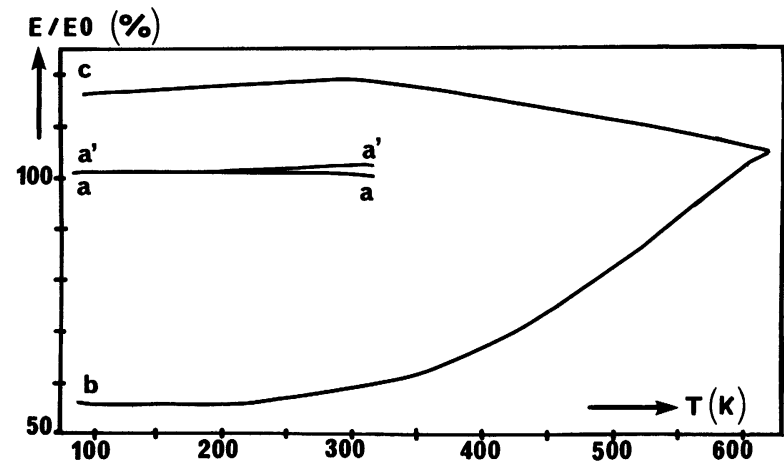

Fig. 1. - Evolution du module d'Young avec l'état structural du matériau : - brut d'élaboration (a : désaimanté; $\mathrm{a}^{\prime}$ : aimanté); - relaxé structuralement à $620 \mathrm{~K}$ (b : désaimanté; c : aimanté).

[Young's modulus variations as a function of structural state of the material. As received state (a : in demagnetized state; $\mathbf{a}^{\prime}$ : in magnetized state); after structural relaxation (b : in demagnetized state; $\mathrm{c}:$ in magnetized state).] 


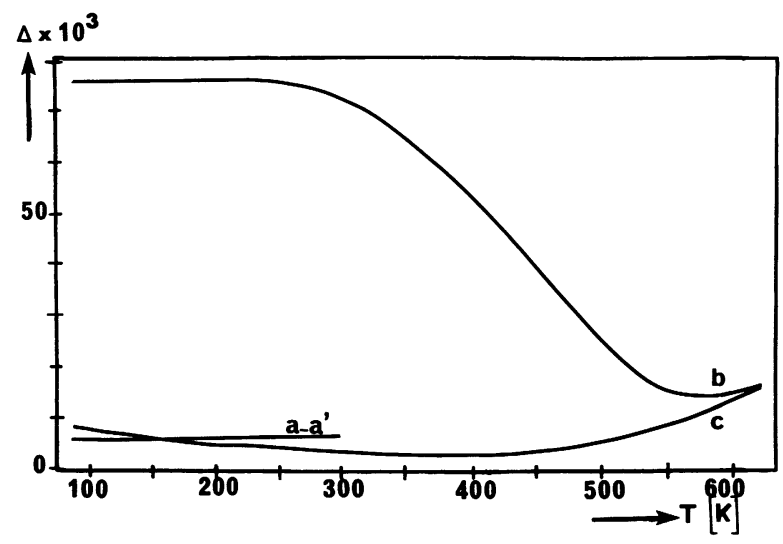

Fig. 2. - Evolution du frottement intérieur avec l'état structural du matériau : a), a), b), c) (idem Fig. 1).

[Internal friction spectrum as a function of structural state of a FeBSiC sample (a-a'-b-c; see Fig. 1).]

pas suffisant pour inhiber totalement le mouvement des parois de domaines ferromagnétiques, responsable du couplage magnétoélastique. Notons, cependant, que pour les basses températures et pour le même champ magnétique appliqué de $3200 \mathrm{~A} / \mathrm{m}$, aucun effet $\Delta E$ mesurable n'est observé.

La comparaison des courbes $\mathrm{a}$ et $\mathrm{b}$ (Figs. 1 et 2 ), relatives à un état désaimanté, montre que la relaxation structurale conduit à une baisse importante du module (effet magnétoélastique) ainsi qu’à une élévation du niveau de frottement intérieur (amortissement magnétomécanique). Ces variations de $E$ et de $\delta$ s'expliquent, comme nous l'avons souligné dans l'introduction, par l'apparition d'une déformation magnétostrictive additionnelle rendue possible par le dépiégeage des parois de Bloch lors de la relaxation structurale. Ces deux effets, particulièrement intenses à basse température (baisse $45 \%$ du module et élévation à $75 \times 10^{-3}$ du frottement intérieur à $80 \mathrm{~K}$ ) décroissent quand la température augmente pour s'annuler au point de Curie $\left(T_{\mathrm{c}}=613 \mathrm{~K}\right)$, en raison de la diminution du coefficient de magnétostriction avec la température [6].

La comparaison entre les courbes $a$ et $b$ ne permet qu'une mise en évidence qualitative de l'effet $\Delta E$ pour un état relaxé structuralement. Pour déterminer une valeur exacte de l'effet magnétoélastique, il est nécessaire d'appliquer un champ magnétique qui, en orientant toutes les parois des domaines ferromagnétiques dans la direction de la contrainte, annihilera tout effet de cette dernière sur leur mouvement et par-là donc, supprimera ce couplage magnétoélastique. C'est ce qui a été réalisé pour les courbes c; la comparaison des courbes $\mathrm{b}$ et $\mathrm{c}$ conduit ainsi à un effet magnétoélastique $\left(E_{\mathrm{s}}-E_{\mathrm{D}}\right) / E_{\mathrm{D}}=0,8$ à $300 \mathrm{~K}$ et à un amortissement magnétomécanique $\delta=70$ $\times 10^{-3}\left(E_{\mathrm{s}}\right.$ : valeur du module correspondant à l'état aimanté à saturation; $E_{\mathrm{D}}$ : à l'état désaimanté). Des valeurs aussi élevées ont rarement été observées.
Seuls Berry et Pritchet [8] ont obtenu un effet $\Delta E$ de valeur comparable avec l'alliage $\mathrm{Fe}_{75} \mathrm{P}_{15} \mathrm{C}_{10}$ en recuisant ce matériau sous champ transversal de manière à orienter les domaines pour avoir un couplage magnétoélastique maximum.

Par ailleurs, la comparaison des courbes a et $\mathrm{c}$ nous permet de préciser l'influence de la relaxation structurale sur les propriétés micromécaniques en s'affranchissant des effets magnétiques. Cette relaxation structurale a donc pour conséquence une augmentation du module d'Young " purement élastique » d'environ $15 \%$ à $300 \mathrm{~K}$ qui traduit un réarrangement structural des atomes dans une configuration énergétiquement plus stable.

Remarquons également que l'on observe (Fig. 1c) une augmentation du module d'Young entre 80 et $300 \mathrm{~K}$, alors que l'on pourrait s'attendre à une décroissance monotone de ce module avec la température pour un matériau aimanté à saturation. Corrélativement, on note une légère augmentation du frottement intérieur quand la température décroît de 300 à $80 \mathrm{~K}$ (Fig. 2c), et la valeur de $\delta$ à $80 \mathrm{~K}$ peut même être supérieure à celle obtenue pour un échantillon brut d'élaboration. Nous reviendrons sur ce point au cours de la discussion.

\subsection{Chargement EN HYDROGÈNE ET INTERACTION PAROIS DE BLOCH-ATOMES D'HYDROGÈNE.}

3.2.1 Etat brut de trempe. - Dans le cas présent d'un échantillon brut d'élaboration et chargé en hydrogène, nous présenterons les résultats obtenus sur le mode fondamental de vibration en relation avec l'état d'aimantation du matériau (désaimanté et aimanté à saturation) et le temps de vieillissement à l'ambiante après chargement en hydrogène.

Les courbes en traits pleins (Fig. 3) relatives à l'état désaimanté montrent la présence d'un pic de frottement intérieur. La largeur à mi-hauteur de ce pic s'étend sur une centaine de degrés, trait caractéristique d'une large distribution des énergies d'activation des atomes d'hydrogène en sites interstitiels.

Après vieillissement à l'ambiante, ce pic de frottement intérieur décroît en amplitude et se translate vers les plus hautes températures. La décroissance du pic semble indiquer une désorption de l'hydrogène. La translation vers les hautes températures peut s'expliquer de la façon suivante [15] : lors du chargement, les atomes d'hydrogène se placent tout d'abord sur les sites de plus basses énergies. Lors de la désorption, les atomes d'hydrogène quitteront en premier lieu les sites de plus haute énergie, d'où une translation vers les hautes températures en fonction du temps de vieillissement à l'ambiante. Ces résultats sont en accord avec ceux de Berry et Pritchet [13] et de Yoshinari et al. [12].

Les courbes de frottement intérieur, obtenues en soumettant l'échantillon à un champ magnétique de $3200 \mathrm{~A} / \mathrm{m}$ (courbes en pointillés, Fig. 3) suivent la même évolution en fonction du temps de vieillissement 


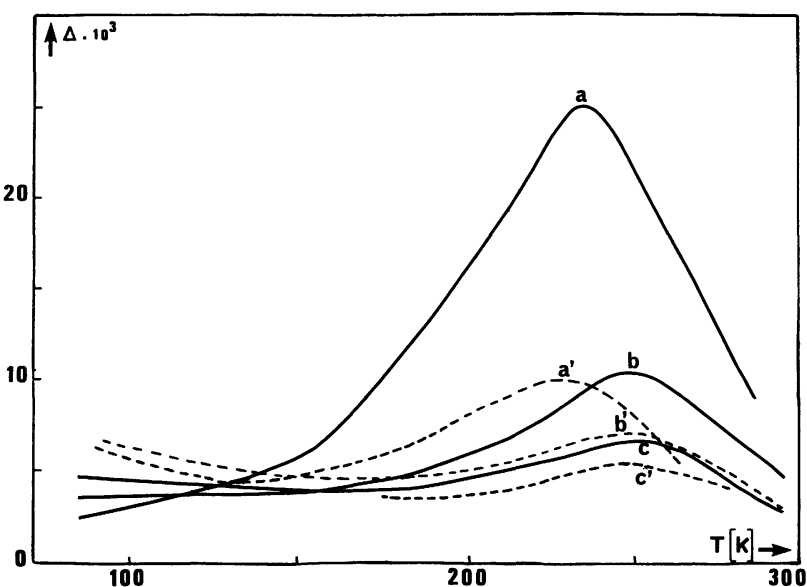

Fig. 3. - Spectre de frottement intérieur du FeBSiC brut d'élaboration, chargé en hydrogène : $a, a^{\prime}:$ immédiatement après chargement ( $a$ : désaimanté; $a^{\prime}$ : aimanté). $b, b^{\prime}$ : après chargement et vieillissement de $24 \mathrm{~h}$ à température ambiante ( $b$ : désaimanté; $b^{\prime}$ : aimanté). $c, c^{\prime}$ : après chargement et vieillissement de $48 \mathrm{~h}$ à température ambiante (c : désaimanté; $c^{\prime}$ : aimanté).

[Internal friction spectrum of an hydrogen charged $\mathrm{FeBSiC}$ sample in as received state. a-a' $\mathbf{a}^{\prime}$ directly after hydrogen permeation $\left(a:\right.$ in demagnetized state; $a^{\prime}:$ in magnetized state); b- $b^{\prime}: 24 \mathrm{~h}$ aging time at room temperature after hydrogen permeation $\left(b:\right.$ in demagnetized state; $b^{\prime}:$ in magnetized state); $c-c^{\prime}: 48 \mathrm{~h}$ aging time at room temperature after hydrogen permeation (c : in demagnetized state; $\mathbf{c}^{\prime}$ : in magnetized state).]

à l'ambiante, mais le fait important est la différence significative relevée entre les niveaux de frottement intérieur dans l'état aimanté et dans l'état désaimanté.

Remarquons d'ores et déjà que cette différence, pouvant atteindre des valeurs de $14 \times 10^{-3}$ directement après chargement en hydrogène et à la température du pic, peut difficilement être attribuée à une interaction hydrogène-parois. En effet, nous avons vu précédemment que le couplage magnétoélastique était très faible dans le cas d'un échantillon brut d'élaboration.

Notons par ailleurs qu'en présence d'un champ magnétique, le frottement intérieur augmente très sensiblement lorsque la température diminue de $150 \mathrm{~K}$ à $80 \mathrm{~K}$ (Figs. 3a' et $\mathrm{b}^{\prime}$ ). Alors qu'en l'absence d'hydrogène, ce phénomène n'était observé qu'à l'état relaxé, il apparaît ici à l'état brut d'élaboration.

Par ailleurs, nous avons évalué l'énergie d'activation correspondant au phénomène de relaxation des atomes d'hydrogène et ce, en travaillant sur trois harmoniques différents, allant de 160 à $2000 \mathrm{~Hz}$ (Fig. 5). Nous obtenons une énergie d'activation $Q=0,55 \pm 0,05 \mathrm{eV}$ correspondant au phénomène de relaxation des atomes d'hydrogène immédiatement après chargement, résultat en bon accord avec ce que Berry et Pritchet observent sur le metglas 2826. Notons qu'une valeur approchée de l'énergie d'acti-

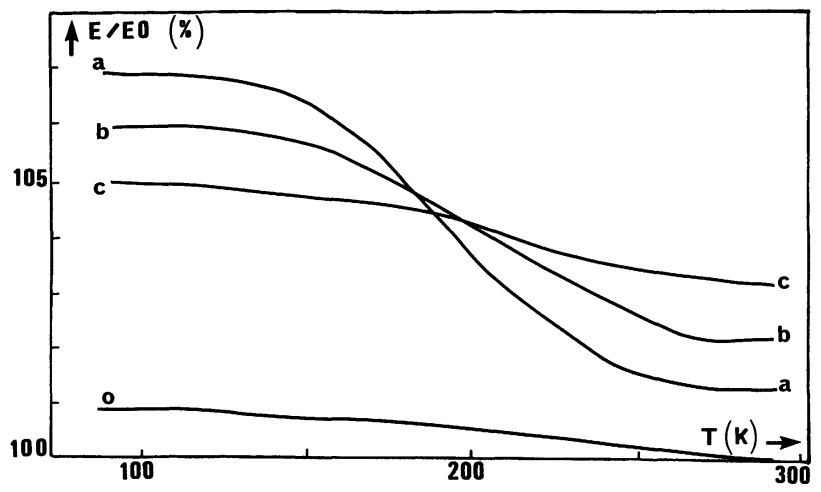

Fig. 4. - Influence de l'hydrogène sur le module d'Young du FeBSiC brut d'élaboration : 0 : avant chargement; $\mathrm{a}:$ directement après chargement; $\mathrm{b}:$ après chargement et vieillissement de $24 \mathrm{~h}$ à température ambiante; $\mathrm{c}$ : après chargement et vieillissement de $48 \mathrm{~h}$ à température ambiante.

[Young's modulus variations versus temperature in the case of an hydrogen charged $\mathrm{FeBSiC}$ sample in as received state. $\mathrm{o}$ : before hydrogen charging; a : directly after hydrogen permeation; $b: 24 \mathrm{~h}$ aging time at room temperature after hydrogen permeation; c : $48 \mathrm{~h}$ aging time at room temperature after hydrogen permeation.]

vation après un vieillissement de $24 \mathrm{~h}$ à l'ambiante a pu être obtenue (Fig. 5) et qu'elle corrobore le fait que l'hydrogène quitte d'abord les sites de plus hautes énergies.

Les courbes donnant la variation du module d'Young avec la température (Fig. 4) présentent trois parties caractéristiques. A basse température $(80 \mathrm{~K})$, le temps de relaxation des atomes d'hydrogène est très grand devant la période de sollicitation de l'éprou-

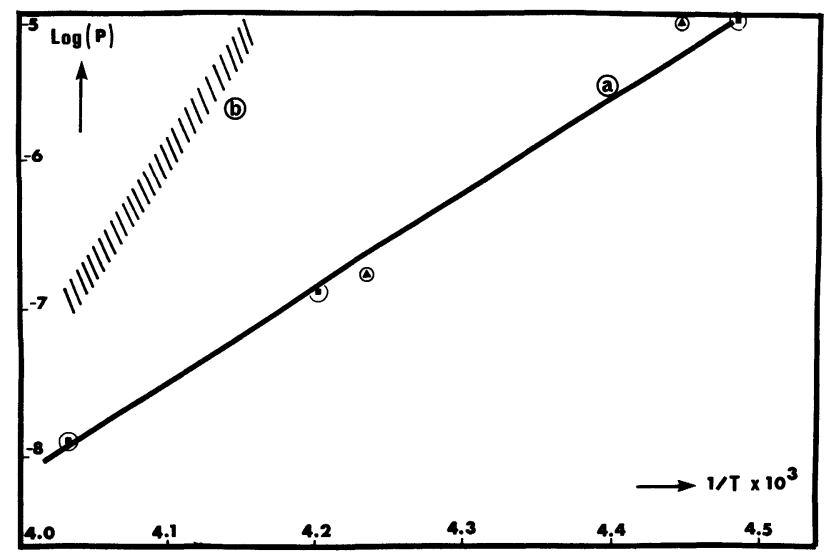

Fig. 5. - Energie d'activation du processus de relaxation des atomes d'hydrogène. $a$ : directement après chargement en hydrogène; $\mathrm{b}$ : évaluation, vieilli $24 \mathrm{~h}$ à température ambiante après chargement.

[Activation energy of hydrogen atoms relaxation phenomenon. a : directly after hydrogen permeation; $b$ : evaluation, $24 \mathrm{~h}$ aging time at room temperature after hydrogen permeation.] 
vette et cela se traduit donc par une augmentation du module liée à la présence d'hydrogène " non mobile " au sein du matériau. Après vieillissement à l'ambiante, nous remarquons à $80 \mathrm{~K}$ une baisse de module causée par la désorption de l'hydrogène, résultat qui est bien corrélé avec les mesures de frottement intérieur qui montrent une décroissance de la hauteur du pic.

Dans le domaine de température correspondant au phénomène de relaxation des atomes d'hydrogène, nous observons une anomalie du module, effet qui va en s'amenuisant au cours du vieillissement.

A température ambiante, le module d'Young est significativement plus faible qu'à $80 \mathrm{~K}$. En effet, les atomes d'hydrogène, fortement mobiles à température ambiante, n'ont plus ou peu d'incidence sur la valeur du module élastique. Par contre, alors que l'hydrogène atomique ne participe pas à cette température à une rigidification du matériau, on note, après vieillissement à l'ambiante, une augmentation du module d'Young, ce qui est contraire à l'idée de désorption (voir discussion $\S 4$ ).

Remarquons également que la valeur du module d'Young a $80 \mathrm{~K}$ après vieillissement reste supérieure de l'ordre de 3 à $4 \%$ à celle correspondant à l'état brut d'élaboration non chargé (Fig. 4c).

3.2.2 Etat relaxé structuralement. - Les résultats obtenus sur un échantillon relaxé structuralement et chargé en hydrogène sont présentés sur les figures 6 (frottement intérieur) et 7 (module d'Young).

Après relaxation structurale à $620 \mathrm{~K}$ ayant pour résultat un effet magnétoélastique important, l'échantillon est chargé électrolytiquement en hydrogène. La conséquence d'un tel traitement est l'apparition immédiatement après chargement, d'un pic de frottement intérieur d'amplitude équivalente à celle obtenue dans le cas d'un ruban brut d'élaboration (Fig. 6c). Mais, après chargement en hydrogène et vieillissement de l'échantillon à $300 \mathrm{~K}$, on observe une augmentation de la hauteur du pic (Fig. 6d). Le pic va même jusqu'à dépasser (Fig. 6e) le niveau de frottement intérieur correspondant à l'état relaxé non chargé (Fig. 6b). Lorsque le vieillissement à l'ambiante est prolongé, la hauteur du pic rediminue (Fig. 6f).

Si l'échantillon relaxé structuralement et chargé en hydrogène est soumis à un champ magnétique (Fig. 8), on observe la même évolution du pic de frottement intérieur que pour un échantillon brut d'élaboration (Fig. 4). Ceci montre donc que le phénomène d'augmentation puis de diminution de la hauteur du pic dans un état désaimanté en fonction du temps de vieillissement à l'ambiante (Fig. 6), trouve son origine dans une interaction entre atomes d'hydrogène et parois des domaines ferromagnétiques.

Cette interaction est par ailleurs mise en évidence en observant les variations du module (Fig. 7) et du frottement intérieur (Fig. 6) à $80 \mathrm{~K}$. En effet, nous remarquons, après chargement en hydrogène, une disparition quasi totale de l'effet magnétoélastique (Fig. 7) et de l'amortissement magnétomécanique
(Fig. 6), résultant du piégeage des parois de Bloch Après maintien à l'ambiante, l'effet $\Delta E$ se redéveloppe (le module d'Young diminue, Fig. 7d) ainsi que l'amortissement magnétomécanique (le frottement intérieur augmente), conséquence du dépiégeage partiel de ces parois.

Afin de s'assurer si un recouvrement total de l'effet magnétoélastique et de l'amortissement magnétomécanique est possible, un recuit in situ à $600 \mathrm{~K}$ est réalisé après un vieillissement de $72 \mathrm{~h}$ à l'ambiante dans le but de faciliter la désorption de l'hydrogène introduit dans le matériau. L'amortissement magnétomécanique reste alors plus faible d'environ $30 \times 10^{-3}$ par

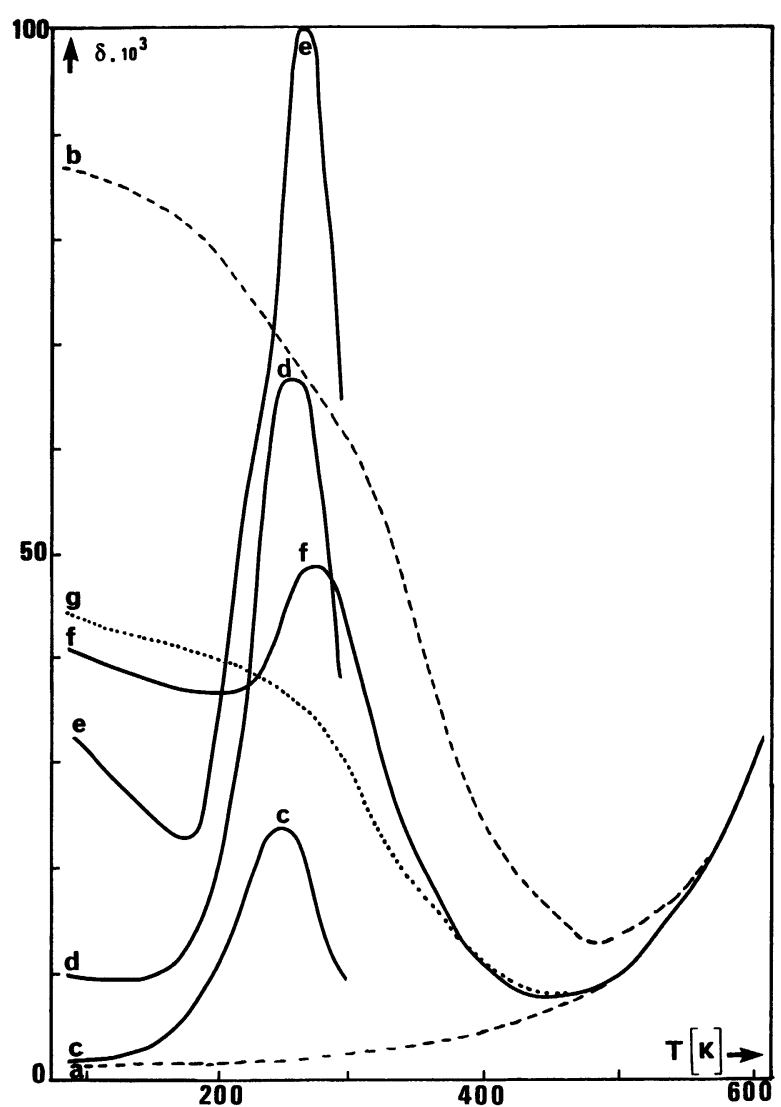

Fig. 6. - Influence du vieillissement après chargement en hydrogène sur le spectre de frottement intérieur du FeBSiC relaxé structuralement. a : état brut d'élaboration, avant chargement; b : état relaxé, avant chargement; $c$ : directement après chargement en hydrogène; $d:$ après vieillissement $24 \mathrm{~h}$ à température ambiante; $\mathrm{e}:$ après vieillissement $48 \mathrm{~h}$ à température ambiante; $\mathrm{f}:$ après vieillissement $72 \mathrm{~h}$ à température ambiante; $\mathrm{g}: \mathrm{f}+$ montée à $600 \mathrm{~K}$.

[Internal friction spectrum of a structurally relaxed FeBSiC sample as a function of temperature. a : in as received state and before hydrogen charging; $\mathrm{b}$ : after structural relaxation and before hydrogen charging; $\mathrm{c}$ : directly after hydrogen permeation; $\mathrm{d}: 24 \mathrm{~h}$ aging time at room temperature after hydrogen permeation; e : $48 \mathrm{~h}$ aging time at room temperature after hydrogen permeation; $\mathrm{f}: 72 \mathrm{~h}$ aging time at room temperature after hydrogen permeation; $\mathrm{g}$ : last heating up to $600 \mathrm{~K}$ following $\mathrm{f}$ measurements.] 


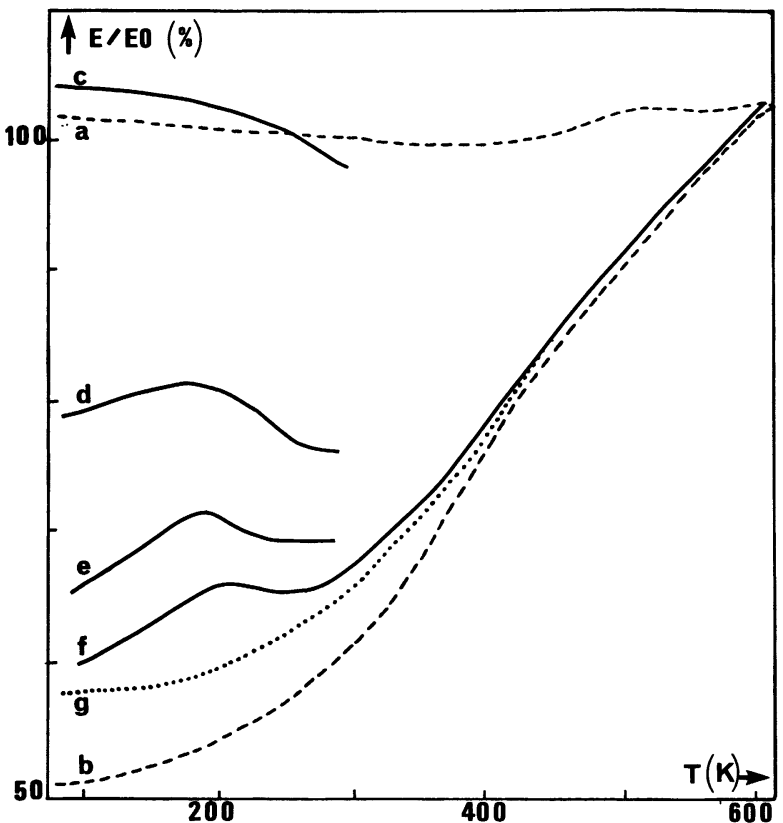

Fig. 7. - Influence du vieillissement après chargement en hydrogène sur le module d'Young du FeBSiC relaxé structuralement : a-b-c-d-e-f-g : idem figure 7.

[Young's modulus variations of a structurally relaxed FeBSiC sample as a function of temperature. a-b-c-d-e-f-g : see figure 6.]

rapport à l'état relaxé avant chargement (Fig. 6g). De même le module reste plus élevé d'environ $10 \%$ à $80 \mathrm{~K}$ (Fig. 7g). Le recouvrement de l'effet magnétoélastique n'est donc pas total.

\section{Discussion.}

Les résultats expérimentaux obtenus par mesure de frottement intérieur et de module d'Young font ressortir plusieurs points que nous allons tenter de préciser et d'interpréter :

a) Tout d'abord ces essais ont mis en évidence, sous champ magnétique, un phénomène d'élévation du frottement intérieur aux basses températures, en particulier à l'état relaxé et après chargement en hydrogène.

b) Dans le cas d'un matériau brut d'élaboration, le vieillissement à l'ambiante d'un échantillon chargé en hydrogène entraîne une augmentation du module d'Young. A l'état relaxé, cet effet est masqué par le couplage magnétoélastique; toutefois, on relève après recuit à $600 \mathrm{~K}$, que l'effet magnétoélastique et l'amortissement magnétomécanique ne sont pas totalement recouvrés.

c) Dans le cas d'un échantillon brut d'élaboration chargé en hydrogène, l'application d'un champ magnétique diminue de manière significative la hauteur du pic de frottement intérieur associé à la relaxation des atomes d'hydrogène. Pour un échantillon relaxé

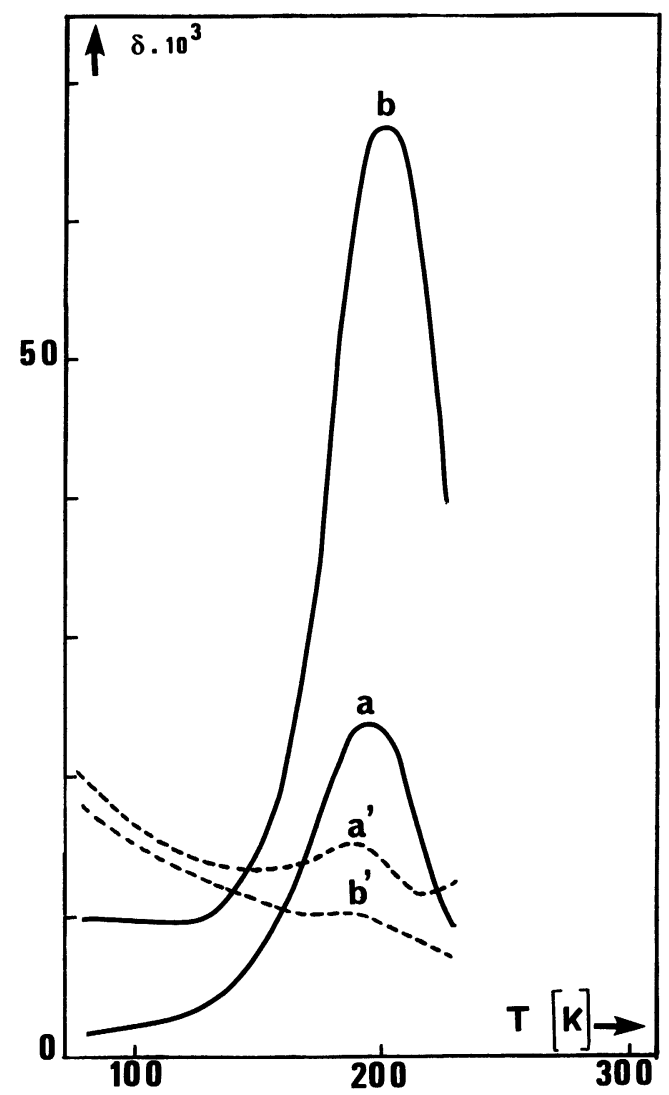

Fig. 8. - Influence du champ magnétique sur le spectre de frottement intérieur d'un échantillon relaxé structuralement : $\mathrm{a}-\mathrm{a}^{\prime}$ : directement après chargement $(\mathrm{a}:$ désaimanté ; $a^{\prime}$ : aimanté); $b-b^{\prime}$ : après vieillissement de $24 \mathrm{~h}$ à température ambiante $\left(b\right.$ : désaimanté; $b^{\prime}$ : aimanté).

[Magnetic field dependence of a structurally relaxed sample internal friction spectrum. a-a' : directly after hydrogen permeation $\left(a:\right.$ in demagnetized state; $a^{\prime}:$ in magnetized state); b-b' : $24 \mathrm{~h}$ aging time at room temperature after hydrogen permeation $\left(b:\right.$ in demagnetized state; $b^{\prime}:$ in magnetized state).]

structuralement et chargé en hydrogène, une interaction hydrogène-parois importante est par ailleurs mise en évidence.

\subsection{CONSÉQueNCE DE L'APPLICATION D'UN CHAMP} MAGNÉTIQUE SUR LES VALEURS DU MODULE D'YOUNG ET DU FROTTEMENT INTÉRIEUR À BASSE TEMPÉRATURE. Dans le cas d'un échantillon brut d'élaboration, les parois de domaine sont très peu mobiles du fait du taux de contraintes internes élevé. Il en résulte donc, à température ambiante, un effet $\Delta E$ faible. A basse température si nous admettons que le mouvement des parois est thermiquement activé, ces parois seront encore moins mobiles qu'à température ambiante, d'où un effet $\Delta E$ négligeable (Figs. 1a et a').

Dans le cas d'un échantillon relaxé structuralement, le champ appliqué de $3200 \mathrm{~A} / \mathrm{m}$ est suffisant pour saturer magnétiquement le matériau au-dessus de la température ambiante alors qu'au-dessous, ce n'est 
plus le cas, d'où la subsistance d'un effet $\Delta E$ et l'observation d'une légère augmentation du module entre 80 et $300 \mathrm{~K}$.

En ce qui concerne le frottement intérieur, dans le cas d'un échantillon brut d'élaboration, nous n'observons pas de différence significative entre les états désaimanté et aimanté (Figs. 2a et a') et le frottement intérieur reste quasiment constant entre $80 \mathrm{~K}$ et $300 \mathrm{~K}$. Par contre, pour un échantillon relaxé structuralement et/ou en présence d'hydrogène au sein du matériau, l'application d'un champ magnétique a pour conséquence une augmentation de $\delta$ quand la température décroît de 150 à $80 \mathrm{~K}$ (Figs. 2, 3 et 8 ).

Afin de comprendre ces phénomènes, nous avons suivi l'évolution du module d'Young avec le champ magnétique appliqué. En toute rigueur, l'aimantation à saturation devrait correspondre à la valeur du champ pour laquelle le module devient constant. Un tel comportement n'est pas toujours observé du fait que, une fois la saturation atteinte, peut apparaître un " effet de pôle » [16]. Cet effet de pôle se caractérise par une augmentation apparente du module variant linéairement avec le champ magnétique appliqué, augmentation d'autant plus importante que la fréquence de vibration est faible. Néanmoins nous avons pu constater que :

a) plus la température est basse, plus il est difficile d'aimanter à saturation nos échantillons. Cette constatation est en bon accord avec les observations de Berry et Pritchet sur un metglas 2826 [9], ainsi que celles de S. M. Bhagat et al. sur un metglas 2826 B [17];

b) en présence d'hydrogène, le champ à appliquer pour aboutir à la saturation est plus élevé que pour le matériau non chargé, fait également mis en évidence par Berry et Pritchet [11]. L'élévation de frottement intérieur à basse température, à champ constant, peut donc être la conséquence d'une aimantation relativement plus faible du matériau, c'est-à-dire plus éloignée de la valeur de l'aimantation à saturation.

Ces remarques peuvent conduire à une explication de l'élévation de frottement intérieur à basse température en terme d'évolution de l'effet $\Delta E$ et donc de l'amortissement magnétomécanique avec l'état de saturation du matériau.

Pour expliciter cette hypothèse, rappelons que l'effet magnétoélastique peut se décomposer en trois contributions : macroscopique, microscopique et hystérétique [10]. La composante due aux courants de Foucault macroscopiques prend en compte l'aimantation globale de l'échantillon. Elle est nulle pour un champ nul et un champ saturant et passe donc par un maximum pour une aimantation intermédiaire. La composante due aux courants de Foucault microscopiques, relative à l'aimantation au niveau de chaque domaine, est strictement décroissante avec le champ. Toutes deux sont fonction de la fréquence de vibration. La composante hystérétique, quant à elle, fonction de l'amplitude de vibration et passant également par un maximum pour une aimantation intermédiaire a une incidence plus faible sur l'effet $\Delta E$ total que les deux autres composantes. Le fait de saturer plus difficilement quand la température décroît de 300 à $80 \mathrm{~K}$, et en présence d'hydrogène, nous conduit alors, pour un champ magnétique constant de $3200 \mathrm{~A} / \mathrm{m}$, à faire intervenir la relaxation par courants de Foucault macroscopiques. L'intervention de cette composante permet alors d'expliquer qu'à basse température, le frottement intérieur à l'état aimanté puisse être supérieur à celui de l'état désaimanté (Figs. 3 et 8). De plus, nous constatons que l'élévation du frottement intérieur à basse température va en augmentant quand la température diminue. En effet, la saturation devenant plus difficile, il en résulte une augmentation de la composante de relaxation par courants de Foucault macroscopiques.

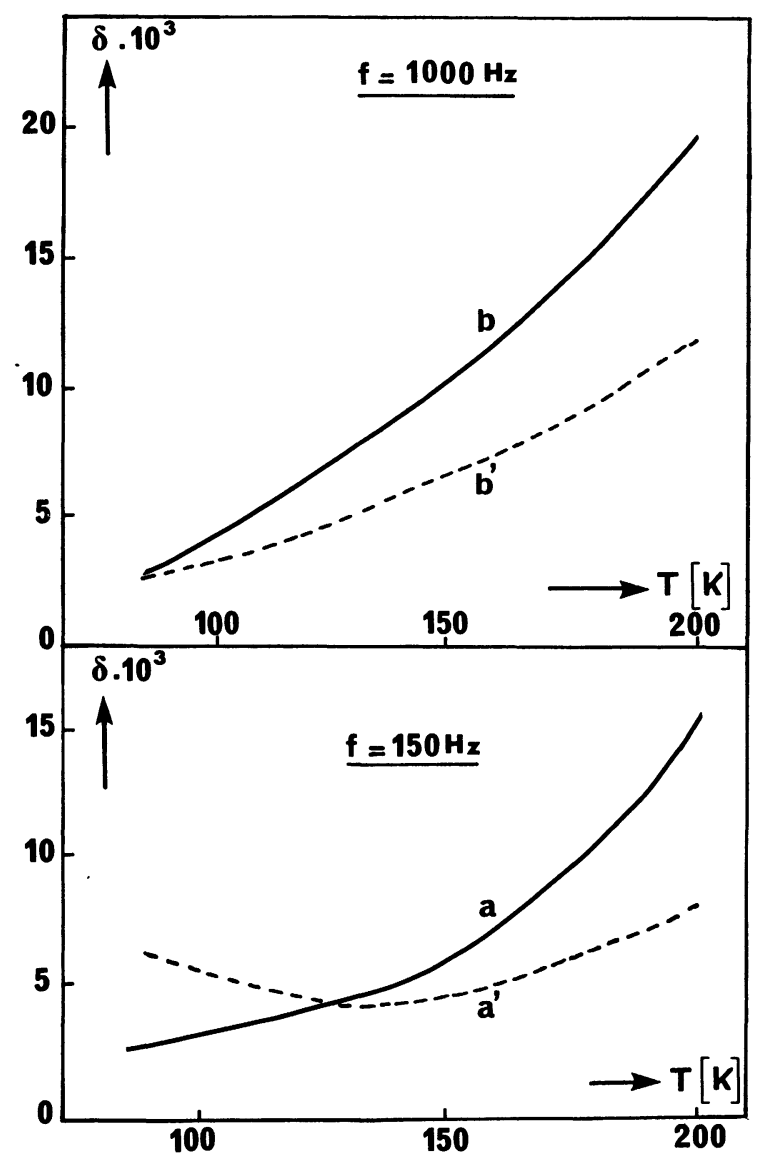

Fig. 9. - Spectre de frottement intérieur entre 80 et $200 \mathrm{~K}$ d'un échantillon brut d'élaboration, chargé en hydrogène : $a-b$ : directement après chargement, à l'état désaimanté; $\mathrm{a}^{\prime}-\mathrm{b}^{\prime}$ : directement après chargement, à l'état aimanté. $\left(\mathrm{a}-\mathrm{a}^{\prime}: f=150 \mathrm{~Hz}\right.$; b-b' $: f=1000 \mathrm{~Hz}$.)

[Internal friction spectrum between 80 and $200 \mathrm{~K}$ of an hydrogen charged sample in as received state. $a-b$ : directly after hydrogen permeation ( $a:$ in demagnetized state; $a^{\prime}$ : in magnetized state); $a^{\prime}-b^{\prime}: 24 \mathrm{~h}$ aging time at room temperature after hydrogen permeation $(b:$ in demagnetized state; $\mathrm{b}^{\prime}:$ in magnetized state). (a-a' $: f=150 \mathrm{~Hz}$; $\mathrm{b}^{\mathrm{b}} \mathrm{b}^{\prime}: f=1000 \mathrm{~Hz}$.)] 
Par ailleurs, ce'phénomène d'élévation de frottement intérieur à basse température et à l'état aimanté n'apparaît plus pour une fréquence plus élevée (Fig. 9). Cela semble montrer que la fréquence de vibration a une influence sur la saturation du matériau. Ces résultats peuvent être rapprochés de ce qu'observent Hernando et al. sur du metglas 2826 [18]. Il faut remarquer égalelement que la déformée de la lame vibrante est différente pour les deux modes de vibration. L'amplitude de vibration est ainsi plus élevée à $150 \mathrm{~Hz}$ qu'à $1000 \mathrm{~Hz}$, ce qui favorise la composante hystérétique et peut donc contribuer à ce phénomène d'élévation du frottement intérieur à basse température.

Notons enfin qu'il serait tentant d'attribuer un phénomène qui disparaît quand la fréquence augmente, et en présence d'un champ magnétique, à un effet de pôle. Cependant, celui-ci n'a aucune raison d'apparaître uniquement aux basses températures, et, de plus, une saturation plus difficile va à l'encontre de l'existence d'un effet de pôle.

4.2 DÉSORPTION-RECOMBINAISON DE L'HYDROGÈNE. Dans le cas d'un échantillon brut d'élaboration, le module d'Young mesuré à la température ambiante augmente en fonction du temps de vieillissement. Cette rigidification du matériau peut être la conséquence soit d'une diffusion vers l'intérieur du matériau et/ou d'une recombinaison d'une partie des atomes d'hydrogène peut être sous forme d'hydrures, soit d'une évolution microstructurale irréversible associée au phénomène de sorption-désorption de l'hydrogène. Le fait que pour un échantillon relaxé structuralement, le couplage magnétoélastique n'est que partiellement recouvré après un maintien à l'ambiante et même après recuit à $600 \mathrm{~K}$ (Figs. 6 et 7), peut s'expliquer de la même manière.

N. Moser et al. [19] envisagent un effet inverse pour expliquer leurs résultats de traînage magnétique sur un verre $\mathrm{Co}_{75} \mathrm{Si}_{15} \mathrm{~B}_{10}$ brut d'élaboration : ils attribuent l'augmentation du pic de réluctivité après recuit entre $260 \mathrm{~K}$ et $350 \mathrm{~K}$ à la dissociation d'amas d'hydrogène existant initialement après chargement, peut être sous forme d'hydrures. Pour des recuits à des températures supérieures à $330 \mathrm{~K}$, ils observent ensuite une disparition du pic qu'ils attribuent à une désorption de l'hydrogène. Les hypothèses avancées par $\mathrm{N}$. Moser et al. [19] ne permettent pas d'expliquer nos résultats. En effet, une dissociation d'amas d'hydrogène entraînerait une augmentation du nombre des atomes d'hydrogène participant à la relaxation à $200 \mathrm{~K}$ et donc une augmentation du pic de frottement intérieur; c'est l'inverse qui est observé. En outre, une désorption de l'hydrogène au-dessus de $350 \mathrm{~K}$ conduirait à une rediminution du module d'Young.

Notons enfin que des mesures combinées de traînage magnétique et de frottement intérieur ont conduit $\mathrm{P}$. Moser et al. [20] à expliquer le pic de frottement intérieur observé sur du $\mathrm{Fe}_{40} \mathrm{Ni}_{40} \mathrm{P}_{14} \mathrm{~B}_{6}$ et du $\mathrm{Fe}_{80} \mathrm{~B}_{20}$ en terme de "d.d.l.d." (" dislocation dipole like defects ") interagissant avec l'hydrogène. Cette notion de d.d.l.d. pourrait s'accorder avec nos résultats dans la mesure où ces "défauts " agiraient comme pièges pour les atomes d'hydrogène ou comme sites de germination d'hydrures.

4.3 INFLUENCE D'UN CHAMP MAGNÉTIQUE SUR LA RELAXATION DES ATOMES D'HYDROGÈNE ET INTERACTION HYDROGÈNE-PAROIS. - Dans le cas d'un échantillon brut d'élaboration, l'amplitude du pic de frottement intérieur diminue par application d'un champ magnétique. L'effet magnétoélastique étant faible dans ce cas, il est difficile d'interpréter cette diminution du pic par un phénomène d'interaction hydrogène-parois. Il est toutefois possible d'envisager que l'aimantation du matériau induit, du fait de la faible mobilité des parois et de la nature magnétostrictive du verre métallique, une contrainte statique dont la conséquence serait une redistribution ou réorientation des atomes d'hydrogène. Il en résulterait donc une diminution (sous l'effet du champ) du nombre des entités participant au phénomène de relaxation mécanique.

Dans le cas d'un échantillon relaxé structuralement, le pic de frottement intérieur observé sur un matériau ferromagnétique et magnétostrictif, tel que le FeBSiC, chargé en hydrogène, peut se décomposer en la somme de deux contributions, l'une due à la relaxation des atomes d'hydrogène et l'autre associée à l'amortissement d'origine magnétomécanique qui révèle une interaction importante entre les parois de domaines et les atomes d'hydrogène (Fig. 6). Cette double action de l'hydrogène sur le frottement intérieur a été illustrée pour un échantillon relaxé structuralement sur la figure 8. La relaxation des atomes d'hydrogène conduit à une contribution continûment décroissante avec le temps de vieillissement après chargement. La contribution d'origine magnéto-mécanique passe, elle, par un maximum pour un temps de vieillissement de $48 \mathrm{~h}$ à $300 \mathrm{~K}$. Pour interpréter ce dernier phénomène, il est possible de faire l'analogie avec le " peaking effect» observé dans les solides cristallins [21] et qui est le résultat de l'évolution de la concentration d'agents piégeants interstitiels sur la mobilité des dislocations après irradiation. Dans notre cas, l'agent piégeant se trouve être l'hydrogène et les parois de domaines ferromagnétiques sont les entités mobiles.

Immédiatement après chargement en hydrogène, les parois de domaines sont piégées, d'où un couplage magnétoélastique faible. Lors du vieillissement, entraînant le départ d'hydrogène, le mouvement des parois devient plus facile sans être, en tout état de cause en phase avec la contrainte appliquée : le déphasage passe alors par un maximum. Cela se caractérise par une élévation importante du niveau de frottement intérieur (Fig. 7e), pouvant ainsi atteindre des valeurs supérieures au niveau obtenu avec un matériau sans hydrogène, après relaxation structurale. Lorsque la désorption est plus avancée, le piégeage des parois devient moins effectif, leur mouvement est de plus en 
plus aisé et le déphasage entre contrainte et déformation va diminuer : cela peut ainsi expliquer la diminution du pic de frottement intérieur après un temps de désorption important (Fig. 7f).

Ce phénomène d'interaction hydrogène-parois, est également mentionné par Berry et Pritchet sur un metglas 2605 [11], mais ces auteurs se sont contentés d'observer l'augmentation du pic après un vieillissement de $24 \mathrm{~h}$ à l'ambiante.

\section{Conclusion.}

Il ressort de cette étude que le frottement intérieur et le module d'Young sont deux grandeurs particulièrement adaptées pour suivre la relaxation structurale d'un verre métallique ferromagnétique et magnétostrictif ainsi que son comportement vis-à-vis de la présence d'hydrogène. En particulier, la très grande sensibilité de l'effet magnétoélastique à l'état structural du matériau et à l'état de chargement en hydrogène permet de réaliser une spectroscopie micromécanique du matériau.

Les mesures effectuées ont mis en évidence :

- un couplage magnétoélastique important après relaxation structurale du Fe-B-Si-C (effet $\Delta E=0,8$ et amortissement magnétomécanique $\delta$ pouvant atteindre $\left.8 \times 10^{-2}\right)$;
- une augmentation du module observé à l'état brut d'élaboration ainsi qu'un non-recouvrement total de l'effet $\Delta E$ et de l'amortissement magnétomécanique, après recuit à $600 \mathrm{~K}$ d'un échantillon relaxé et chargé en hydrogène, montrant que soit une partie de l'hydrogène introduit reste au sein du matériau, peut-être sous forme d'hydrures ou peut-être à l'état atomique dans un puits de potentiel profond, soit que l'hydrogène a provoqué des modifications microstructurales irréversibles lors du cycle sorption-désorption ;

- une redistribution ou réorientation des atomes d'hydrogène sous l'effet du champ se caractérisant par une baisse du pic de frottement intérieur d'un échantillon brut d'élaboration après aimantation à saturation;

- une interaction importante entre les parois de domaines ferromagnétiques et les atomes d'hydrogène, l'évolution du pic de frottement intérieur en fonction du temps de vieillissement à $300 \mathrm{~K}$ après chargement mettant en évidence un phénomène analogue au « peaking effect » observé dans les matériaux cristallins.

\section{Remerciements.}

Ce travail a pu être réalisé grâce à une participation financière du centre de recherches de Pont-à-Mousson S.A.

Bibliographie

[1] Barmatz, M., Chen, H. S., Phys. Rev. B 9 (1974) 4073.

[2] Chen, H. S., Leamy, H. J., Barmatz, M., J. Non Cryst. Solids 5 (1971) 444.

[3] Kursomovic, A., Scott, M. G., Girt, E., CahN, R. W., Scripta Met. 14 (1980) 1303.

[4] Bothe, K., Neuhauser, N., J. Non Cryst. Solids 56 (1983) 279.

[5] Morito, N., Mat. Sci. Eng. 60 (1983) 261.

[6] Guyot, F., Fouquet, F., Perez, J., J. Physique 44 (1983) 139.

[7] GuYOT, F., Thèse D.I., Lyon (1982).

[8] BERRY, B. S., Elastic and Inelastic Behavior in Metallic Glasses (American Society for metals) 1976.

[9] Berry, B. S., Pritchet, W. C., A.I.P. Conf. Proc. (New-York) 34 (1976).

[10] Berry, B. S., Pritchet, W. C., J. Appl. Phys. 47 (1976) 3295.

[11] Berry, B. S., Pritchet, W. C., J. Appl. Phys. 52 (1981) 1865.

[12] Yoshinari, O., KoIwa, M., InOUe, R., Masumoto, T., Acta. Met. 12 (1983) 2063.
[13] Berry, B. S., Pritchet, W. C., Scripta Met. 15 (1981) 637.

[14] Chambron, W., Lancon, F., Chamberod, A., Scripta Met. 15 (1984) 29.

[15] Agyeman, K., Armbruster, E., Kunzi, H. U., Das Gupta, A., Guntherodt, M. J., J. Physique Colloq. 42 (1981) C5-535.

[16] Berry, B. S., Pritchet, W. C., J. Appl. Phys. 50 (1979) 1630.

[17] Bhagat, S. M., Spano, M. L., J. Appl. Phys. 50 (1979) 1580.

[18] Hernando, A., Maduerga, V., Barandiaran, J. M., Liniers, M., J. Magn. Magn. Mat. 28 (1982) 109.

[19] Moser, N., Kronmuller, H., Phys. Lett. 93A (1982) 101.

[20] Moser, P., Chi-Mei Mo, Moumene, M., J. Physique Colloq. 44 (1983) C9-91.

[21] Minier, C., Lauzier, J., Esnouf, C., Fantozzi, G., J. Physique Colloq. 44 (1983) C9-51. 